\title{
Studies on amino acids admixtured triglycine sulphophosphate crystals
}

\author{
A.S. Haja Hameed ${ }^{\mathrm{a}}$, G. Ravi ${ }^{\mathrm{b}}$, C.W. Lan ${ }^{\mathrm{a}, *}$ \\ ${ }^{a}$ Department of Chemical Engineering, National Taiwan University, No. 1, Sec. 4, Rossevelt Rd., Taipei 10617, Taiwan ROC \\ ${ }^{\mathrm{b}}$ Advanced Materials Laboratory, National Institute for Materials Science(NIMS), Tsukuba, Ibaraki 305-0044, Japan
}

Available online 21 December 2004

\begin{abstract}
To study the improved characteristics of triglycine sulphophosphate (TGSP) crystals, TGSP crystals mixed with amino acids have been grown by temperature reduction method. Amino acids like alanine, valine and asparagine were selected for doping. The influence of amino acids in the crystal lattice and molecular vibrational frequencies of various groups in the samples have been studied using X-ray powder diffraction and Fourier transform infrared (FTIR) analyses. The thermal behaviour of pure and amino mixed TGSP crystals was studied from the DT and TG analyses. (C) 2004 Elsevier B.V. All rights reserved.
\end{abstract}

PACS: 81.10.Dn; 81.70.Pg

Keywords: A1. Characterization; A2. Growth from solution; B1. Organic compounds; B2. Ferroelectric materials

\section{Introduction}

Triglycine sulphate (TGS) is a well known ferroelectric material and it is widely used in fabricating thermal detectors, vidicons and image systems due to its second-order phase transition at room temperature and higher pyroelectric coefficient as well [1,2]. The major drawback in TGS crystal is the depolarization of glycine molecule which has serious implication in the device performance. To overcome this, inorganic and

\footnotetext{
*Corresponding author. Tel./fax: + 886223633917.

E-mail address: cwlan@ntu.edu.tw (C.W. Lan).
}

organic dopants in TGS have been tried. The doping of L-alanine in TGS (ATGS) solved the problem of depolarization. In ATGS, the presence of extra methyl group in the place of a hydrogen atom resulted in stabilization of the structure [3-6]. The added advantage of doping results in reduced dielectric permittivity and hence the pyroelectric figure of merit was higher than the undoped TGS $[7,8]$. Even though doping enhances the characteristics of TGS, it could not alleviate the proliferation of microbes. Since glycine and cosubstituted amino acids are rich in nutrient and hence it promotes the formation of microbes, thereby reducing their shelf life. In order to 
overcome this microbial problem, the partial substitution of phosphate in TGS was tried and it gives rise to enhanced characteristics and shelf life time of the solution. The phosphate incorporated TGS (TGSP) crystal belongs to monoclinic system with space group of $2 \mathrm{P}_{1}$ in the ferroelectric phase. The lattice parameter values of TGSP are $a=9.15 \AA, b=12.69 \AA$ and $c=5.73 \AA$ [9]. It has a wide ferroelectric plane and offers the possibility of fabricating wider single element detectors [10]. It was also observed that the dielectric permittivity of TGSP was reduced as compared to pure TGS due to the extra proton available for charge compensation in TGSP. The effect of 1-tyrosine and l-cystine on TGS crystal was recently reported $[11,12]$. The aim of the present work is to study the contribution of amino acids on the TGSP characteristics. The amino acids - alanine, valine and asparagine were separately mixed in three TGSP solutions and their crystals were grown. For the grown crystals, X-ray powder diffraction, Fourier transform infra red (FTIR) spectrum analysis, DTA and TGA studies have been carried out.

\section{Experimental procedure}

Triglycine sulphophosphate (TGSP) was synthesized according to the following chemical reaction:

$$
\begin{gathered}
3\left(\mathrm{NH}_{2} \mathrm{CH}_{2} \mathrm{COOH}\right)+x\left(\mathrm{H}_{2} \mathrm{SO}_{4}\right)+(1-x)\left(\mathrm{H}_{3} \mathrm{PO}_{4}\right) \\
\quad \rightarrow\left(\mathrm{NH}_{2} \mathrm{CH}_{2} \mathrm{COOH}\right)_{3} \cdot\left(\mathrm{H}_{2} \mathrm{SO}_{4}\right)_{x} \cdot\left(\mathrm{H}_{3} \mathrm{PO}_{4}\right)_{1-x},
\end{gathered}
$$

where $x$ was varied from 0 to 1 in steps of 0.25 . Due to the aggregation of tiny polycrystals at higher molar concentration of orthophosphoric acid in TGS mother solution, the growth was restricted up to $50 \%$ of $\mathrm{H}_{2} \mathrm{SO}_{4}$ and $50 \%$ of $\mathrm{H}_{3} \mathrm{PO}_{4}$. For the synthesis of amino acids mixed TGSP, $20 \mathrm{~mole} \%$ of alanine, valine and asparagine were admixtured separately in the TGSP solutions. After fully evaporating water, dried salts of pure and amino mixed TGSP were obtained and used for the growth. After saturating the solutions, filtration was done using $0.2 \mu \mathrm{m}$ porosity nuclear filter after due preheating. Twenty milliliter of each solution was poured into four perti-dishes and allowed to nucleate spontaneously at room tem- perature so as to get transparent seeds for the growth runs. To grow optical quality and strainfree bulk crystals, the seeds obtained were tested under optical microscope. These seeds were suspended in the respective saturated solutions kept at $40{ }^{\circ} \mathrm{C}$ and then the solution temperature was reduced with a cooling rate of $0.12{ }^{\circ} \mathrm{C} /$ day.

The cleaved plates from the grown crystals of pure and amino acids mixed TGSP were powdered well. The X-ray powder diffraction pattern was recorded by using Rich Seifert diffractometer. The powdered samples were subjected to monochromatic X-rays of wavelength $1.5418 \AA\left(\mathrm{CuK}_{\alpha}\right)$ at a scan speed of $2^{\circ} / \mathrm{min}$. Differential thermal (DTA) and thermo gravimetric analyses (TGA) were also carried out for the samples using STA 1500 thermal analyzer with a heating rate of $5{ }^{\circ} \mathrm{C} / \mathrm{min}$. $\mathrm{KBr}$ pellet technique was employed to record FTIR spectra using Bruker model FS $66 \mathrm{~V}$ spectrometer. The FTIR spectra were taken in the range of $400-4400 \mathrm{~cm}^{-1}$ for these samples.

\section{Results and discussion}

\subsection{Growth and plane identification}

Pure and amino acids mixed TGSP crystals are shown in Fig. 1, which were grown by low temperature solution technique for a period of 25 days. Growth rates along three crystallographic axes for the grown crystals are presented in Table 1. It was found that the pure and amino acids mixed TGSP crystals are having the faster growth rate along the $b$-axis. Among all these crystals, alanine mixed TGSP was found to have enhanced growth along the three axes. Valine mixed crystals possessed less growth as compared with other amino family of crystals. The asparagine molecule in TGSP enhances the growth along the $b$-axis as compared to pure and valine mixed TGSP crystals and its decreased growth rate was observed along the $a$-axis. The pure TGSP crystal possesses (1 $\left.\begin{array}{lll}1 & 0\end{array}\right),\left(\begin{array}{lll}1 & 0 & 1\end{array}\right),\left(\begin{array}{lll}0 & 0 & 1\end{array}\right)$ and $\left(\begin{array}{lll}1 & 1 & 0\end{array}\right)$ faces. The addition of alanine in TGSP causes the decreased area of (100) and (110) faces as compared to (101) face. In valine mixed TGSP crystal, ( $\left.\begin{array}{lll}1 & 0 & 1\end{array}\right)$ face was found to be absent. 

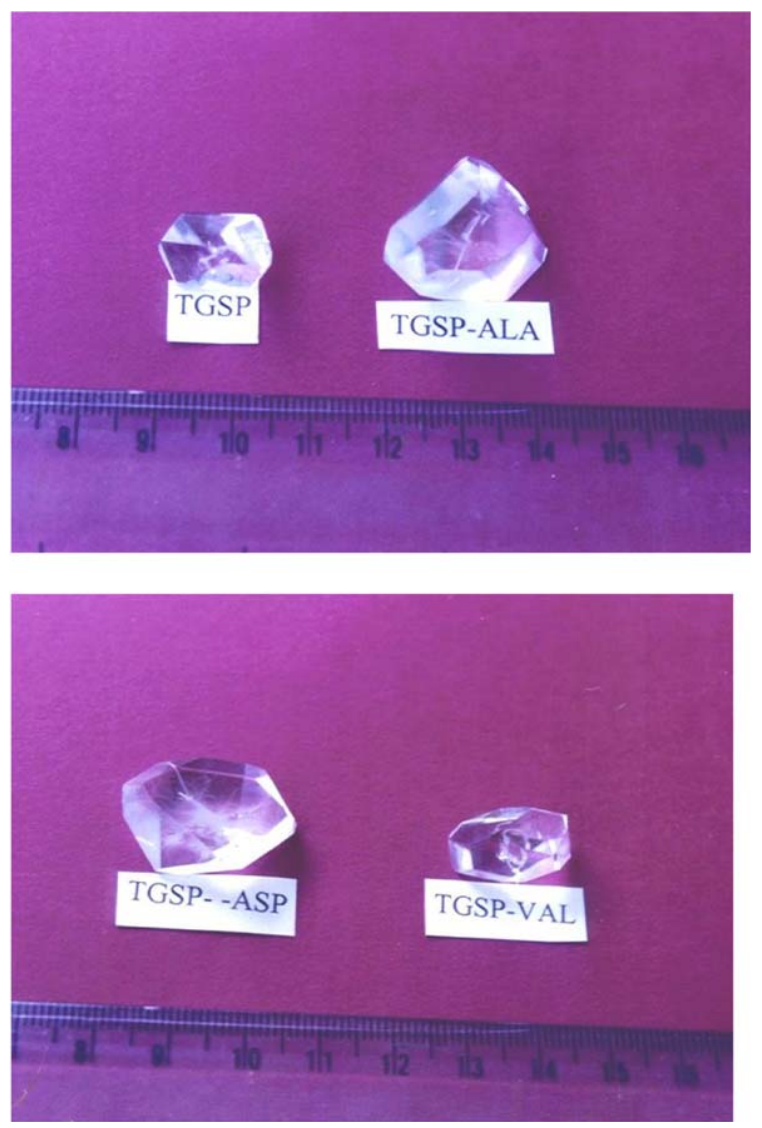

Fig. 1. Photograph of the grown crystals.

Table 1

Growth rates along $a, b$ and $c$-axes of pure and amino acids mixed TGSP crystals

\begin{tabular}{llll}
\hline Sample & \multicolumn{3}{l}{ Growth rate along the axes (mm/day) } \\
\cline { 2 - 4 } & $a$ & $b$ & $c$ \\
\hline TGSP & 0.305 & 0.440 & 0.253 \\
TGSP-ALA & 0.375 & 0.778 & 0.477 \\
TGSP-VAL & 0.249 & 0.477 & 0.313 \\
TGSP-ASP & 0.257 & 0.777 & 0.411 \\
\hline
\end{tabular}

In the case of asparagine mixed crystal, less growth was observed along the $a$-axis and its $\left(\begin{array}{lll}0 & 0 & 1\end{array}\right)$ face has larger area compared to pure TGSP (Table 1).

\subsection{X-ray powder diffraction studies}

It is observed from the powder X-ray diffraction that the addition of amino acids alters the lattice parameter values of pure TGSP crystals. These variations resulted from the minor shifts in peak positions. The calculated lattice parameters are tabulated in Table 2.

\subsection{Thermal studies}

In the earlier literature, thermal studies indicating the decomposition temperature and weight loss of the TGS family crystals were not found. Hence, DTA and TGA were carried out for pure and amino acids mixed TGSP samples. It is observed from DTA curves that the decomposition temperature for pure TGSP starts from 200.6 to $222.6{ }^{\circ} \mathrm{C}$. TG curve shows that TGSP loses its weight by $46.19 \%$. In the case of alanine mixed TGSP crystals, decomposition is delayed by $7{ }^{\circ} \mathrm{C}$ with $3 \%$ greater weight loss as compared to pure TGSP. The starting and final decomposition temperatures and weight loss of valine mixed TGSP crystal are found in between alanine mixed and pure TGSP crystals. No change was observed in initial and final decomposition temperatures for asparagine mixed crystal and it was found that the initial and final decomposition occurred at 201.3 and $222.5^{\circ} \mathrm{C}$ but weight loss was about $50 \%$. The variation of decomposition temperatures and weight loss of pure and asparagine mixed TGSP crystals are shown in Fig. 2.

\subsection{FTIR spectroscopic analysis}

The FTIR spectra were taken in the range of $400-4400 \mathrm{~cm}^{-1}$ for pure and asparagine mixed TGSP crystals. The FTIR spectra of the samples are shown in Fig. 3. A broad strong absorption occurs around $3165 \mathrm{~cm}^{-1}$ due to $\mathrm{OH}$ stretching bands. The asymmetric $\mathrm{NH}_{3}^{+}$and symmetrical bending are observed at 1622 and $1505 \mathrm{~cm}^{-1}$, respectively. The strong absorption observed at $1707 \mathrm{~cm}^{-1}$ is due to the $\mathrm{C}=\mathrm{O}$ stretching of $-\mathrm{COOH}$ group. $\mathrm{C}-\mathrm{N}$ broad band is assigned to $1129 \mathrm{~cm}^{-1}$. The asymmetric 
Table 2

Lattice parameter values of pure and amino acids mixed TGSP crystals

\begin{tabular}{llll}
\hline Sample & \multicolumn{3}{l}{ Lattice parameter values $(\AA)$} \\
\cline { 2 - 4 } & $a$ & $b$ & $c$ \\
\hline TGSP & 9.37 & 12.69 & 5.89 \\
TGSP-ALA & 9.44 & 12.73 & 5.85 \\
TGSP-VAL & 9.44 & 12.78 & 5.88 \\
TGSP-ASP & 9.48 & 12.69 & 5.89 \\
\hline
\end{tabular}
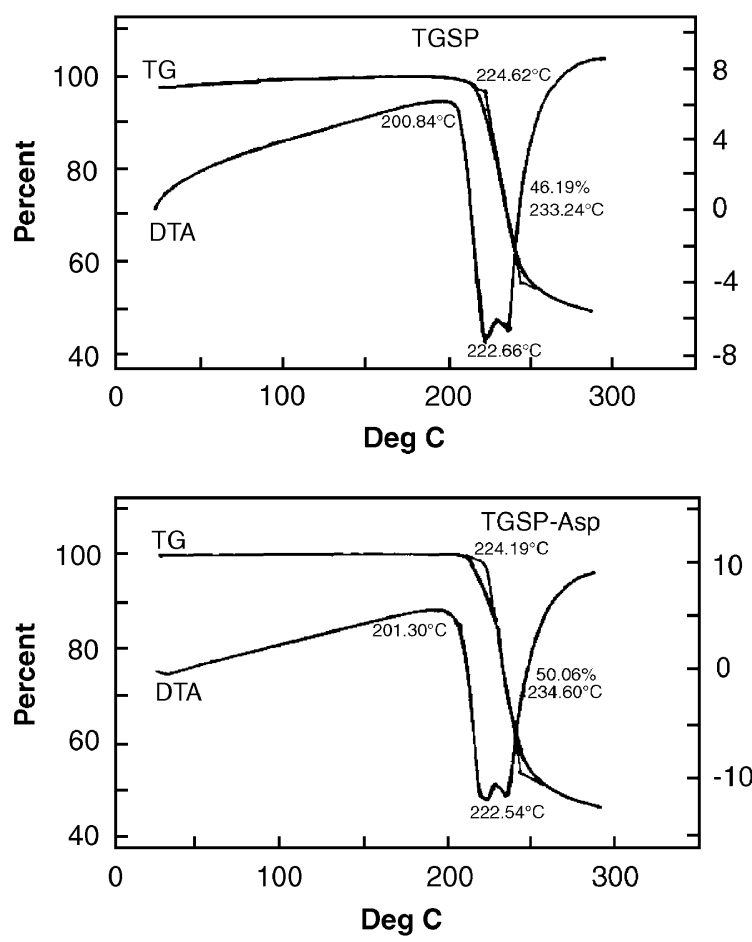

Fig. 2. DT and TG curves for pure and asparagine mixed TGSP crystals.

stretching of both sulphate and phosphate lies in the same envelope at $1017 \mathrm{~cm}^{-1}$ [8]. The influence and incorporation of alanine, valine and asparagine were confirmed from the wavelength assignment of methyl group in the case of alanine, isoprophyl group in the valine mixed TGSP and amide group in the asparagine mixed crystal.
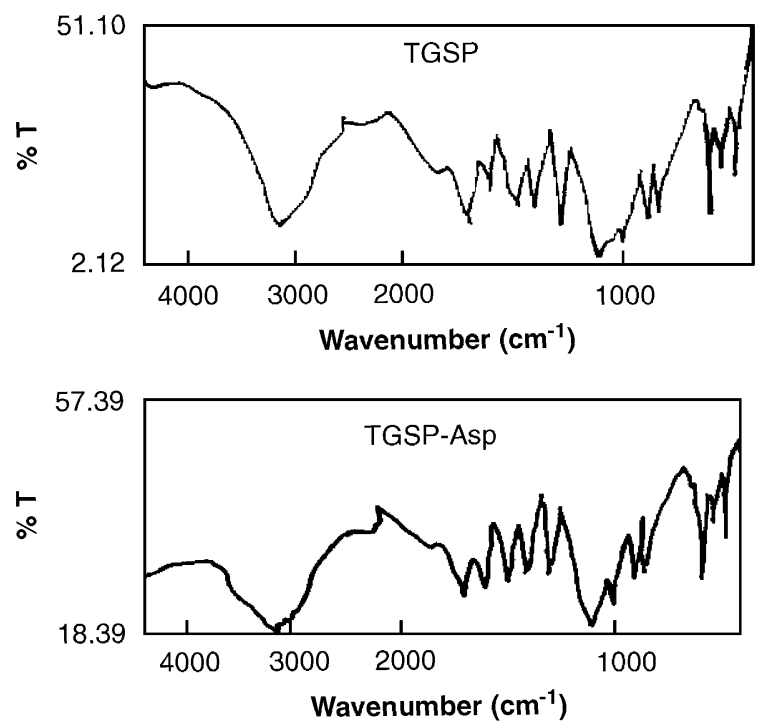

Fig. 3. FTIR spectra for pure and amino asparagine mixed TGSP crystals.

\section{Conclusion}

The pure, alanine, valine and asparagine mixed TGSP crystals were successfully grown. From the morphology of the grown crystals, the variations in area on (1 000$),\left(\begin{array}{llll}1 & 0 & 1\end{array}\right),\left(\begin{array}{lll}0 & 0 & 1\end{array}\right)$ and $\left(\begin{array}{lll}1 & 1 & 0\end{array}\right)$ faces were observed. The growth rates along the three axes of pure and amino mixed TGSP crystals were also found to be varied. These changes are due to the partial substitution of alanine, valine and asparagine in the respective TGSP crystals. Among these, alanine mixed crystals gave a good result in terms of crystal dimension, which was evidenced from the fast growth rate along $b$ and $c$ axes of the crystal. The variation in the lattice parameter values was observed from the X-ray powder diffraction studies and this evidences the incorporation of respective amino acids in TGSP crystal lattice. DTA and TGA curves explained the decomposition temperature and weight loss of the materials during heating. In these, alanine mixed TGSP crystal delayed the starting decomposition and this may be due to the hardening of lattice. From the FTIR spectra, the various functional groups, symmetric stretching, asymmetric stretching and bending were assigned for the grown crystals. 


\section{References}

[1] H.P. Beerman, IEEE Trans. Devices 16 (1968) 554.

[2] E.H. Putley, Semicond. Semimet. 5 (1970) 259.

[3] C.S. Fang, X.I. Yao, A.S. Bhalla, L.E. Cross, Ferroelectrics 51 (1983) 9.

[4] K.L. Bye, P.W. Whipps, E.T. Keve, Ferroelectrics 4 (1972) 253.

[5] A.S. Bhalla, C.S. Fang, Y. Xi, L.E. Cross, Appl. Phys. Lett. 43 (1983) 932.

[6] V.A. Borisenok, T.R. Volk, A.S. Koshelev, E.Z. Noviskii, S.A. Shramchenko, L.A. Shuvalov, Sov. Phys. Crystallogr. 33 (1988) 780.
[7] G. Ravi, S. Anbukumar, C. Subramanian, P.K.C. Pillai, P. Ramasamy, Ferroelectrics 166 (1995) 47.

[8] G. Arunmozhi, R. Jayavel, C. Subramanian, Mater. Chem. Phys. 50 (1997) 57.

[9] G. Ravi, S. Anbukumar, P. Ramasamsy, J. Crystal Growth 133 (1993) 212.

[10] G. Ravi, S. Anbukumar, P. Ramasamy, Mater. Chem. Phys. 37 (1994) 180.

[11] K. Meera, S. Aravazhi, P. Santhanaraghavan, P. Ramasamy, J. Crystal Growth 211 (2000) 220.

[12] K. Meera, R. Muralidharan, P. Santhanaraghavan, R. Goplakrishnan, P. Ramasamy, J. Crystal Growh 226 (2001) 303. 